\title{
Associations of 2D speckle tracking echocardiography-based right heart deformation parameters and invasively assessed hemodynamic measurements in patients with pulmonary hypertension
}

Lena Theres $^{1,2^{*}}$ (D) Anne Hübscher ${ }^{1}$, Karl Stangl ${ }^{1,2}$, Henryk Dreger ${ }^{1,2}$, Fabian Knebel ${ }^{1,2,3}$, Anna Brand ${ }^{1,2+}$ and Bernd Hewing 1,2,3,4,5+

\begin{abstract}
Background: We aimed to evaluate associations of right atrial (RA) and right ventricular (RV) strain parameters assessed by 2D speckle tracking echocardiography (2D STE) with invasively measured hemodynamic parameters in patients with and without pulmonary hypertension $(\mathrm{PH})$.

Methods: In this study, we analyzed 78 all-comer patients undergoing invasive hemodynamic assessment by left and right heart catheterization. Standard transthoracic echocardiographic assessment was performed under the same hemodynamic conditions. RA and RV longitudinal strain parameters were analyzed using 2D STE. PH was defined as invasively obtained mean pulmonary arterial pressure (mPAP) $\geq 25 \mathrm{mmHg}$ at rest and was further divided into pre-capillary PH (pulmonary capillary wedge pressure [PCWP] $\leq 15 \mathrm{mmHg}$ ), post-capillary PH $(\mathrm{PCWP}>15$ $\mathrm{mmHg}$ ) and combined PH (PCWP > $15 \mathrm{mmHg}$ and difference between diastolic PAP and PCWP of $\geq 7 \mathrm{mmHg}$ ). Correlation analyses between variables were calculated with Pearson's or Spearman's correlation coefficient as applicable.

\footnotetext{
* Correspondence: lena.theres@charite.de

${ }^{\dagger}$ Anna Brand and Bernd Hewing share senior authorship

${ }^{1}$ Medizinische Klinik m.S. Kardiologie und Angiologie,

Charité-Universitätsmedizin, Campus Mitte, Charitéplatz 1, 10117 Berlin, Germany

${ }^{2}$ DZHK (German Center for Cardiovascular Research), partner site, Berlin, Germany

Full list of author information is available at the end of the article
}

(c) The Author(s). 2020 Open Access This article is licensed under a Creative Commons Attribution 4.0 International License, which permits use, sharing, adaptation, distribution and reproduction in any medium or format, as long as you give appropriate credit to the original author(s) and the source, provide a link to the Creative Commons licence, and indicate if changes were made. The images or other third party material in this article are included in the article's Creative Commons licence, unless indicated otherwise in a credit line to the material. If material is not included in the article's Creative Commons licence and your intended use is not permitted by statutory regulation or exceeds the permitted use, you will need to obtain permission directly from the copyright holder. To view a copy of this licence, visit http://creativecommons.org/licenses/by/4.0/ The Creative Commons Public Domain Dedication waiver (http://creativecommons.org/publicdomain/zero/1.0/) applies to the data made available in this article, unless otherwise stated in a credit line to the data. 


\begin{abstract}
(Continued from previous page)
Results: Out of 78 patients, 45 presented with PH. Within the PH group, 39 had post-capillary, five had combined pre- and post-capillary $\mathrm{PH}$, and one had pre-capillary PH. Patients with $\mathrm{PH}$ had a significantly increased RA area (PH $22.0 \pm 9.2 \mathrm{~cm}^{2}$, non-PH $\left.17.3 \pm 10.7 \mathrm{~cm}^{2} ; p=0.003\right)$ and end-systolic RV area $\left(\mathrm{PH} 14.7 \pm 6.1\right.$, non-PH $11.9 \pm 4.8 \mathrm{~cm}^{2}$; $p=0.022)$. RV mid strain was significantly reduced in $\mathrm{PH}(\mathrm{PH}-17.4 \pm 7.8$, non- $\mathrm{PH}:-21.6 \pm 5.5 ; p=0.019)$. Average peak systolic RA strain (RAS) and average peak systolic RV strain (RVS) showed a significant association with mPAP $(r=-0.470, p=0.001$ and $r=0.490, p=0.001$, respectively $)$ and with PCWP $(r=-0.296, p=0.048$ and $r=0.365, p=$ 0.015 , respectively) in patients with PH. Furthermore, RV apical, mid and basal strain as well as RV free wall strain showed moderate associations with mPAP. In patients without PH, there were no associations detectable between RA or RV strain parameters and MPAP and PCWP.
\end{abstract}

Conclusion: In an all-comer cohort, RA and RV strain parameters showed significant associations with invasively assessed mPAP and PCWP in patients with predominantly post-capillary PH. These associations may be useful in clinical practice to assess the impact of post-capillary PH on myocardial right heart function.

Keywords: Right atrial strain, Right ventricular strain, Pulmonary hypertension, 2D speckle tracking echocardiography, Post-capillary pulmonary hypertension, Hemodynamic

\section{Introduction}

While diagnosis of $\mathrm{PH}$ is based on invasive hemodynamic measurements of pulmonary artery pressure (PAP) by right heart catheterization, transthoracic echocardiography (TTE) remains the widely available screening and monitoring tool for $\mathrm{PH}$. According to current $\mathrm{PH}$ guidelines [1], estimation of systolic PAP is recommended in patients with tricuspid regurgitation by Doppler Imaging. Recommendations, however, on functional assessment of RA and RV impairment due to PH remain scarce. Recent studies have shown that RA and RV dysfunction are important prognostic factors and indicators for adverse outcome in patients with pre-capillary $\mathrm{PH}[2-5]$. 2D STE reportedly constitutes a feasible additional tool for the assessment of RA and RV function [6, 7] and normative reference data for RA and RV 2D STE have been published for healthy populations [8-10]. Furthermore, the prognostic value of RA and RV strain parameters has been evaluated in patients with pre-capillary PH $[11,12]$. However, studies assessing the association of RA and RV mechanics with invasively obtained parameters in patients with $\mathrm{PH}$ remain sparse and exist primarily for pre-capillary $\mathrm{PH}[4$, 13, 14]. Thus, this study aimed to evaluate associations of RA and RV strain values assessed by 2D STE with invasively measured hemodynamic parameters in patients $\mathrm{PH}$ from an all-comer cohort of a cardiology department.

\section{Methods}

\section{Study design and population}

This study is a retrospective analysis of a single-center database of the Cardiology Department, Charité-Universitätsmedizin Berlin, Campus Mitte, Germany. 78 patients were enrolled in the study in the years 2013 to 2015. All underwent hemodynamic assessment by left and right heart catheterization and had a comprehensive TTE examination within $24 \mathrm{~h}$ periprocedural (median
$3.2 \mathrm{~h}$ ). A comprehensive medical history was collected in all study participants by clinical interview at admission and by medical records. Exclusion criteria were ongoing mechanical ventilation, presence of severe mitral valve stenosis, mechanical prosthetic heart valves, heart transplantation, and insufficient image quality. $\mathrm{PH}$ was defined as an invasively measured $\mathrm{mPAP}$ of $\geq 25 \mathrm{mmHg}$ at rest [1]. We distinguished between pre-capillary $\mathrm{PH}$, which is defined as an elevated mPAP of $\geq 25 \mathrm{mmHg}$ but normal PCWP $\leq 15 \mathrm{mmHg}$, and post-capillary $\mathrm{PH}$, where $\mathrm{mPAP}$ and PCWP are both elevated (mPAP $\geq 25 \mathrm{mmHg}$ and PCWP $>15 \mathrm{mmHg}$ ) [1]. Combined pre- and postcapillary PH was defined as an elevated PCWP and diastolic pressure gradient (defined as the difference between diastolic PAP and PCWP) of $\geq 7 \mathrm{mmHg}$ [1].

The study has been approved by the institutional ethics committee of the Charité-Universitätsmedizin Berlin (EA4/067/13) and complied with the Declaration of Helsinki.

\section{Echocardiographic examination}

A comprehensive TTE was performed using a Vivid 7 Dimension or Vivid E9 (GE Vingmed, Horton, Norway; M4S or M5S $1.5-4.5 \mathrm{MHz}$ transducer) ultrasound systems. All investigators in the echocardiography laboratory were blinded for invasively obtained data. Standard left and right heart echocardiographic parameters were obtained according to the recommendations of the American Society of Echocardiography (ASE) and European Association of Cardiovascular Imaging (EACVI) $[15,16]$. These included measurement of tricuspid annular plane systolic excursion (TAPSE) with M-Mode and RV peak systolic velocity (RV-S') with Doppler tissue imaging (DTI). Three beats of each view were recorded for offline analysis (EchoPac PC, GE Vingmed). RA area and RV diameter (basal, mid and apico-basal) were 
measured directly from 2D images according to the recommendations of ASE and EACVI $[15,16]$. Endocardial borders of the RV were traced in an apical four-chamber view for detection of end-diastolic and end-systolic RV area (RVED and RVES area, respectively) and RV fractional area change (FAC) was calculated. Left ventricular ejection fraction (LVEF) was calculated using biplane Simpson's method and Auto-EF tool (GE Vingmed) as previously described [17].

\section{D speckle tracking strain analysis}

For longitudinal strain analyses, multiple standard 2D ultrasound images were recorded with a frame rate between 60 and 80 frames per second and digitally stored for offline analysis (EchoPac PC, GE Vingmed). After manual tracing of the endocardial border of the RA, RV and left ventricle (LV) [18, 19], the region of interest (ROI) was semi-automatically determined. The ROI was manually adjusted for optimal tracking quality when necessary. For all strain analyses, an arithmetic mean value of three individual measurements was used.

For phasic RA strain assessment, a RV-focused four chamber view, avoiding foreshortening of the RA, was chosen. Phasic RA strain analyses were performed using 2D speckle tracking echocardiography [19] identifying strain values during the three phases of the RA cycle from the resulting strain curves (see Fig. 1 for illustration). The maximum excursion of the average strain curve (dotted line in Fig. 1) symbolizes the maximum longitudinal strain and RA reservoir function (RAS). Next, RA conduit function was calculated from the difference of strain values of conduit phase in early diastole (onset of the atrial contraction) and RAS, while RA contraction function was derived from the difference between the endpoint of conduit phase (at the onset of atrial contraction) and the peak negative RA strain shortening (RA contraction, see Fig. 1) [19]. In patients with atrial fibrillation, RA conduit and contractile function was not obtained.

For longitudinal RV strain analyses, a RV-focused apical four-chamber view was used. We analyzed RV strain in four segments, three of them being part of the RV free wall (basal, mid and apical segment) as well as the interventricular septum (see Fig. 2 for illustration). Combining the average peak systolic strain of all RV segments resulted in an average RV strain (RVS), while RV free wall comprises the average strain of all three free wall segments.

LV global longitudinal peak systolic strain (LV-GLPS) was measured semi-automatically by strain analyses of the apical four-, three- and two-chamber-view as previously described [17].

\section{Cardiac catheterization}

All patients had a clinical indication for a combined right and left heart catheterization. Cardiac catheterization measurements were performed as previously described [17]. Briefly, arterial and venous accesses were established by inserting sheaths in the femoral artery and vein, respectively. Then a fluid-filled pigtail catheter (6 French, Cordis, a Cardinal Health company) was placed in the LV for LV end-diastolic pressure (LVEDP) measurements. For measurements of right heart pressures, pulmonary artery pressure (PAP) and PCWP a Corodyn P1 right heart

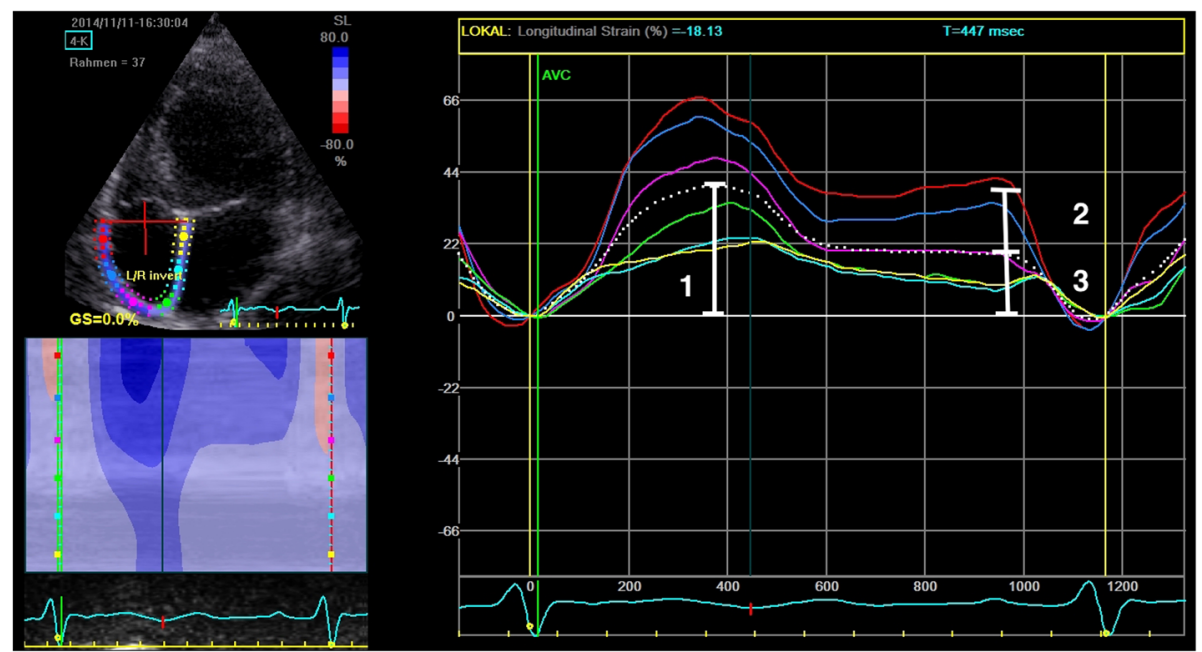

Fig. 1 Representative example of speckle tracking echocardiography (2D STE-) derived longitudinal strain of the right atrium obtained in the apical 4-chamber view. Dotted white curve indicates mean of all segments. The maximum excursion of the average strain curve [1] symbolizes the maximum longitudinal strain and RA reservoir function (RAS). RA conduit function was calculated from the difference between RAS and the end of the conduit phase in early diastole [2] and RA contractile function was derived from the difference between the endpoint of conduit phase and the peak RA contraction [3] 


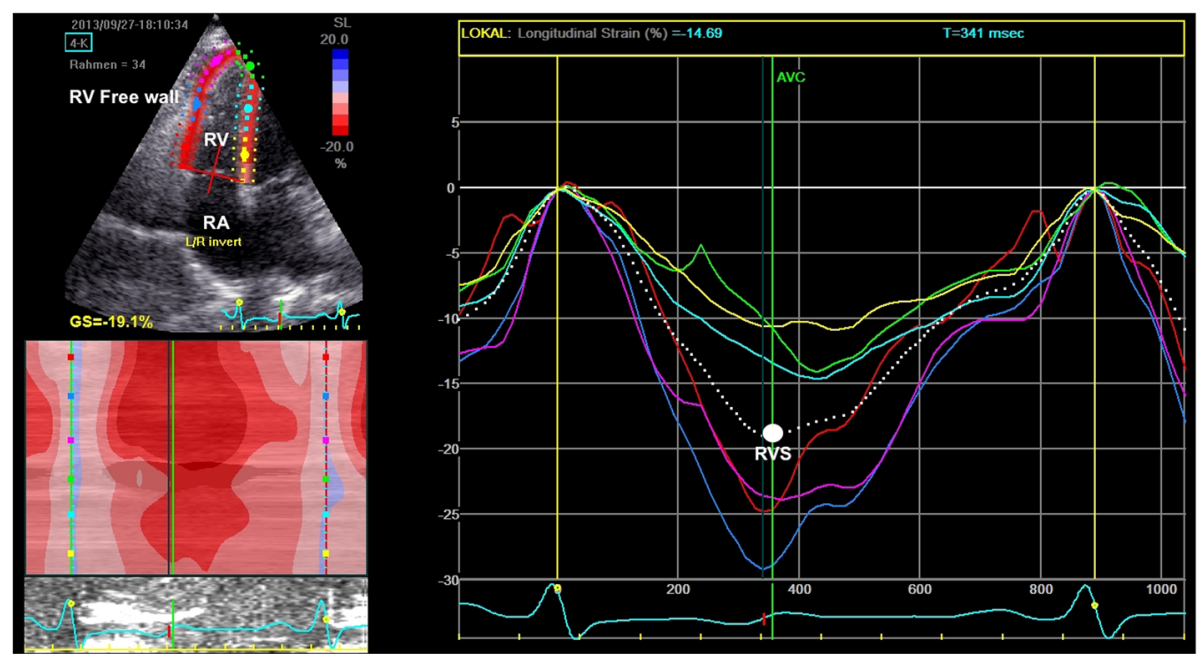

Fig. 2 Representative example of speckle tracking echocardiography (2D STE-) derived longitudinal strain of the right ventricle obtained in the apical 4-chamber view. Dotted white curve indicates mean of all segments, where RVS is derived from maximum excursion. The RV Free wall consists of three segments, which are colored red, dark blue and pink in the STE analysis

catheter (B. Braun, Germany) was used. Exact wedge position was verified by changes in pressure waveforms and fluoroscopy. Cardiac index, systemic vascular resistance (SVR) and pulmonary vascular resistance (PVR) were calculated as previously described [17]. All patients were hemodynamically stable at the time of the examination. All measurements were acquired during an unforced end expiratory breath-hold and were completed before injection of contrast agents into the left ventricle or coronary arteries was performed.

\section{Intra- und inter-observer agreement}

To determine intra- and interobserver variability for RA and RV strain parameters, a second experienced cardiologist, who was blinded to previously obtained data, separately measured RA and RV strain parameters of 20 randomly selected patients. Intraclass correlation coefficients (ICC) were calculated for RVS, RAS, RA conduit and contractile function.

\section{Statistical analysis}

Statistical analysis was performed using SPSS (Version 25, IBM Corporation). For continuous variables, results are expressed as arithmetic mean and standard deviation (SD) or, if not normally distributed variables, as median with interquartile ranges ( $\mathrm{IQR}=25$ th -75 th percentile). Accordingly, Student's t-test or Mann-Whitney U test was used for comparison of groups. Categorical data are presented as absolute numbers and respective percentages. Categorical variables were compared by chi-square tests. For correlation analyses between variables, Pearson's or Spearman's correlation coefficient were calculated as applicable. A $p$-value $<0.05$ was considered statistically significant.

\section{Results}

\section{Study population}

A total of 78 patients were enrolled in the present study. 45 patients presented with $\mathrm{PH}$, while 33 patients had no signs of $\mathrm{PH}$ (non-PH). Within the $\mathrm{PH}$ group, 39 had post-capillary, five had combined pre- and post-capillary $\mathrm{PH}$, and one had pre-capillary PH. Baseline clinical and demographic characteristics of all patients and individual groups of $\mathrm{PH}$ and non-PH patients are listed in Table 1. None of the patients had tricuspid or pulmonary valve stenosis.

\section{Echocardiographic and invasive characteristics}

Detailed cardiac catheterization and echocardiographic data of all patients and individual groups of $\mathrm{PH}$ and non-PH patients are presented in Table 2 . Invasively assessed MPAP, PWCP, and LVEDP were significantly higher in $\mathrm{PH}$ compared to non- $\mathrm{PH}$ patients while cardiac index was significantly lower in the $\mathrm{PH}$ group (Table 2). Patients with PH had significantly larger RA and RV end-systolic area (RVES area) compared to nonPH patients. TAPSE, RV-S', LVEF, and LV-GPLS were significantly lower in the PH group (Table 2).

Due to the presence of atrial fibrillation at examination, assessment of RA conduit and contractile function was limited to 59 patients ( $\mathrm{PH} n=30$, non-PH $n=29$ ). $\mathrm{RA}$ and RV strain was reduced in the $\mathrm{PH}$ group but the difference did not reach statistical significance except for average mid RV strain (Table 3). 
Table 1 Baseline characteristics

\begin{tabular}{|c|c|c|c|c|}
\hline Characteristic & All $(\boldsymbol{n}=78)$ & $\mathrm{PH}(\boldsymbol{n}=45)$ & Non-PH $(\boldsymbol{n}=33)$ & $\boldsymbol{p}$-value \\
\hline Number of patients, n (\%) & 78 & $45(57.7)$ & $33(42.3)$ & \\
\hline Age, years & $72.6 \pm 12.8$ & $75.1 \pm 11.5$ & $69.3 \pm 13.9$ & 0.049 \\
\hline Gender female/male, n (\%) & $17(22.0) / 61$ (78.0) & 16 (35.6) / 29 (64.4) & $32(97.0) / 1(3.0)$ & 0.001 \\
\hline $\mathrm{BMI}, \mathrm{kg} / \mathrm{m}^{2}$ & $26.0(24.0-29.4)$ & $26.7(24.0-29.5)$ & $24.9(24.0-27.8)$ & 0.107 \\
\hline Heart rate, /min & $71.0 \pm 12.3$ & $72.6 \pm 13.4$ & $68.8 \pm 10.2$ & 0.181 \\
\hline $\mathrm{SBP}, \mathrm{mmHg}$ & $137.0(118.7-150.0)$ & $137.0(116.0-149.0)$ & $137.0(121.5-153.5)$ & 0.671 \\
\hline $\mathrm{DBP}, \mathrm{mmHg}$ & $68.4 \pm 12.3$ & $68.9 \pm 13.3$ & $67.8 \pm 11.1$ & 0.693 \\
\hline$A F, n(\%)$ & $21(26.9)$ & $16(35.6)$ & $5(15.2)$ & 0.045 \\
\hline Diabetes, n (\%) & $23(29.5)$ & $17(37.8)$ & $6(18.2)$ & 0.061 \\
\hline Arterial hypertension, n (\%) & $56(71.8)$ & $35(77.8)$ & $21(63.6)$ & 0.170 \\
\hline$C A D, n(\%)$ & $33(42.3)$ & $24(53.3)$ & $9(27.3)$ & 0.021 \\
\hline Previous MI, n (\%) & $13(16.7)$ & $10(22.2)$ & $3(9.1)$ & 0.124 \\
\hline Previous CABG, n (\%) & $7(9.0)$ & $6(13.3)$ & $1(3.0)$ & 0.116 \\
\hline Previous TIA/stroke, n (\%) & $5(6.4)$ & $3(6.7)$ & $2(6.1)$ & 0.914 \\
\hline PAD, n (\%) & $8(10.3)$ & $6(13.3)$ & $2(6.1)$ & 0.296 \\
\hline Previous bAVR, n (\%) & $4(5.1)$ & $3(6.7)$ & $1(3.0)$ & 0.472 \\
\hline PM, n (\%) & $14(17.9)$ & $10(22.2)$ & $4(12.1)$ & 0.251 \\
\hline ICD, n (\%) & $2(2.6)$ & $2(4.4)$ & 0 & 0.220 \\
\hline COPD, n (\%) & $16(20.5)$ & $13(28.9)$ & $3(9.1)$ & 0.032 \\
\hline
\end{tabular}

Data are expressed as mean \pm SD, median with IQR or absolute numbers and respective percentages. AF, atrial fibrillation; BMI, body mass index; SBP, systolic blood pressure; DBP, diastolic blood pressure; CAD, coronary artery disease; MI, myocardial infarction; CABG, coronary artery bypass grafting; TIA, transient ischemic attack; PAD, peripheral artery disease; bAVR, bioprosthetic aortic valve replacement; PM, pace maker; ICD, implantable cardioverter defibrillator; COPD, chronic obstructive pulmonary disease.

\section{Associations between echocardiographic standard and deformation parameters and invasive characteristics}

Associations between echocardiographic and invasively assessed parameters are listed in Table 4. There were no significant associations between echocardiographically assessed right heart parameters and invasively measured mPAP and PCWP in non-PH patients. In $\mathrm{PH}$ patients, RAS showed a significant inverse association with mPAP $(\mathrm{r}=-0.470, p=0.001)$ and with PCWP $(\mathrm{r}=-0.296, p=$ 0.048 ), while RA conduit and contractile function did not show any significant associations with mPAP or PCWP.

Concerning RV parameters, moderate associations between mPAP and RVS $(\mathrm{r}=0.490, \mathrm{p}=0.001)$, RV free wall strain ( $\mathrm{r}=0.466, p=0.002)$, $\mathrm{RV}$ mid $\operatorname{strain}(\mathrm{r}=0.444, p=$ $0.003)$ or RV apical strain $(\mathrm{r}=0.461, \mathrm{p}=0.002)$ were found in $\mathrm{PH}$ patients. Associations between mPAP and TAPSE $(\mathrm{r}=0.378, p=0.010)$, RV-S' $(\mathrm{r}=0.323, p=0.037)$, RVED area $(\mathrm{r}=0.382, p=0.012)$, or RV base diameter $(\mathrm{r}=0.346$, $p=0.023)$ were weak to moderate.

There were significant associations between PCWP and RVED area $(\mathrm{r}=0.389, p=0.019)$, RVES area $(\mathrm{r}=0.338$, $p=0.027)$, RVS $(\mathrm{r}=0.365, p=0.015)$, RV base diameter $(\mathrm{r}=0.318, p=0.038)$, or RV apical strain $(\mathrm{r}=0.348, p=$ 0.220 ). While there was a significant difference in RA area between patients with $\mathrm{PH}$ and without $\mathrm{PH}$, there was no significant association of RA area with mPAP or PCWP.
RVOT VTI and RV-a' showed weak to moderate associations with RAS and RVS (RVOT VTI: $\mathrm{r}=0.244, p=$ 0.042; $\mathrm{r}=-0.306, \mathrm{p}=0.015$ for RAS and RVS; RV-a': $\mathrm{r}=$ $0.405, p=0.001$; and $\mathrm{r}=-0,256, \mathrm{p}=0,055$ for RAS and RVS; respectively). Some of the clinical characteristics with possible impact on $\mathrm{PH}$ diagnosis were different between groups; however, none of these parameters showed significant associations with the diagnosis of $\mathrm{PH}$ in a multivariate logistic regression model (see Supplement, Tables S1 and S2).

\section{Intra- and inter-observer agreement}

The ICC for intra-observer agreement was 0.86 (95\% confidence interval $[\mathrm{CI}]$ 0.68-0.94) for RAS, 0.88 (CI 0.73-0.95) for RA conduit function, 0.86 (CI 0.68-0.94) for RA contractile function and 0.75 (CI 0.48-0.89) for RVS. ICC for inter-observer agreement was 0.92 (CI 0.81-0.97), 0.89 (CI 0.75-0.95), 0.82 (CI 0.61-0.92) and 0.91 (CI 0.8-0.96), respectively.

\section{Discussion}

In the present study, we analyzed echocardiographically and invasively assessed hemodynamic parameters in a cohort of all-comer patients with and without $\mathrm{PH}$. We demonstrated significant associations between right heart deformation parameters (2D STE-derived RA and 
Table 2 Echocardiographic and cardiac catheterization data

\begin{tabular}{|c|c|c|c|c|}
\hline Parameter & All $(\boldsymbol{n}=78)$ & $\mathrm{PH}(\boldsymbol{n}=45)$ & Non-PH $(\boldsymbol{n}=33)$ & $p$-value \\
\hline \multicolumn{5}{|l|}{ Echocardiographic data } \\
\hline RA area, $\mathrm{cm}^{2}$ & $16.9(13.0-24.9)$ & $20.2(15.8-27.0)$ & $14.2(12.3-18.0)$ & 0.003 \\
\hline RVED area, $\mathrm{cm}^{2}$ & $21.9(17.9-25.6)$ & $22.1(18.7-27.3)$ & $20.4(15.8-25.4)$ & 0.130 \\
\hline RVES area, $\mathrm{cm}^{2}$ & $12.2(9.9-15.6)$ & $13.1(10.8-17.1)$ & $10.7(9.3-13.4)$ & 0.022 \\
\hline$F A C, \%$ & $41.3(32.7-49.1)$ & $41.0(33.0-48.4)$ & $43.5(32.3-53.3)$ & 0.749 \\
\hline RV base diameter, $\mathrm{mm}$ & $38.2(33.5-42.9)$ & $39.6(34.6-43.9)$ & $36(30.3-40.7)$ & 0.104 \\
\hline RV mid diameter, mm & $26.6(22.1-32.7)$ & $29.3(22.4-34.6)$ & $24.8(21.5-31.5)$ & 0.146 \\
\hline RV apex-base diameter, mm & $77.4(66.3-84.2)$ & $77.6(66.3-84.9)$ & $75.8(66.4-81.8)$ & 0.402 \\
\hline RVOT mm & $32.3 \pm 4.3$ & $32.5 \pm 4.1$ & $31.8 \pm 4.5$ & 0.54 \\
\hline TAPSE, mm & $19.8 \pm 5.6$ & $17.8 \pm 5.4$ & $22.3 \pm 4.7$ & $<0.001$ \\
\hline$R V-S^{\prime}, \mathrm{cm} / \mathrm{s}$ & $10.9 \pm 3.1$ & $10.0 \pm 3.1$ & $12.2 \pm 2.6$ & 0.003 \\
\hline RVOT VTI & 13.1. \pm 4.0 & $12.0 \pm 4.2$ & $14.6 \pm 3.2$ & 0.006 \\
\hline$R V-e^{\prime} \mathrm{cm} / \mathrm{s}$ & $10.7 \pm 3.3$ & $10.6 \pm 3.8$ & $10.7 \pm 2.7$ & 0.76 \\
\hline $\mathrm{RV}-\mathrm{a}^{\prime} \mathrm{cm} / \mathrm{s}$ & $12.7 \pm 5.6$ & $11.3 \pm 6.4$ & $14.2 \pm 4.3$ & 0.045 \\
\hline LV ejection fraction, \% & $45.0 \pm 13.4$ & $39.0 \pm 12.4$ & $52.4 \pm 11.0$ & $<0.001$ \\
\hline$\geq 55, n(\%)$ & $25(32.1)$ & $8(17.8)$ & $17(51.5)$ & \\
\hline $45-54, n(\%)$ & $17(21.8)$ & $8(17.8)$ & $9(27.3)$ & \\
\hline $30-44, n(\%)$ & $27(34.6)$ & $21(46.7)$ & $6(18.2)$ & \\
\hline$\leq 30, n(\%)$ & $9(11.5)$ & $8(17.8)$ & $1(3.0)$ & \\
\hline LV-GPLS & $-13.6 \pm 5.5$ & $-11.2 \pm 5.3$ & $-16.5 \pm 4.3$ & $<0.001$ \\
\hline Aortic valve regurgitation, n (\%) & & & & 0.122 \\
\hline none & $42(53.8)$ & $20(44.4)$ & $22(66.7)$ & \\
\hline mild & $25(32.1)$ & $16(35.6)$ & $9(27.3)$ & \\
\hline moderate & $9(11.5)$ & $8(17.8)$ & $1(3)$ & \\
\hline severe & $2(2.6)$ & $1(2.2)$ & $1(3)$ & \\
\hline Aortic valve stenosis, n (\%) & & & & 0.544 \\
\hline none & $54(69.2)$ & $29(64.4)$ & $25(75.8)$ & \\
\hline mild & $1(1.3)$ & $1(2.2)$ & 0 & \\
\hline moderate & $1(1.3)$ & $1(2.2)$ & 0 & \\
\hline severe & $22(28.2)$ & $14(31.1)$ & $8(24.2)$ & \\
\hline Mitral valve regurgitation, $\mathrm{n}(\%)$ & & & & 0.002 \\
\hline none & $16(20.5)$ & $8(17.8)$ & $8(24.2)$ & \\
\hline mild & $27(34.6)$ & $9(20.0)$ & $18(54.5)$ & \\
\hline moderate & $30(38.5)$ & $25(55.6)$ & $5(15.2)$ & \\
\hline severe & $5(6.4)$ & $3(6.7)$ & $2(6.1)$ & \\
\hline Mitral valve stenosis, n (\%) & & & & 0.675 \\
\hline none & $75(96.2)$ & $43(95.6)$ & $32(97.0)$ & \\
\hline mild & $2(2.6)$ & $1(2.2)$ & $1(3.0)$ & \\
\hline moderate & $1(1.3)$ & $1(2.2)$ & 0 & \\
\hline Tricuspid valve regurgitation, $\mathrm{n}(\%)$ & & & & $<0.001$ \\
\hline none & $18(23.1)$ & $5(11.1)$ & $13(39.4)$ & \\
\hline mild & $31(39.7)$ & $16(35.6)$ & $15(45.5)$ & \\
\hline moderate & $21(26.9)$ & $16(35.6)$ & $5(15.2)$ & \\
\hline severe & $8(10.3)$ & $8(17.8)$ & 0 & \\
\hline
\end{tabular}


Table 2 Echocardiographic and cardiac catheterization data (Continued)

\begin{tabular}{|c|c|c|c|c|}
\hline Parameter & All $(\boldsymbol{n}=78)$ & $\mathrm{PH}(\boldsymbol{n}=45)$ & Non-PH $(\boldsymbol{n}=33)$ & $\overline{p \text {-value }}$ \\
\hline \multicolumn{4}{|c|}{ Pulmonary valve regurgitation, $\mathrm{n}(\%)$} & \multirow[t]{4}{*}{0.060} \\
\hline none & $50(64.1)$ & $24(53.3)$ & $26(78.8)$ & \\
\hline mild & $27(34.6)$ & $20(44.4)$ & $7(21.2)$ & \\
\hline severe & $1(1.3)$ & $1(2.2)$ & 0 & \\
\hline \multicolumn{5}{|l|}{ Catheterization data } \\
\hline mPAP, mmHg & $28.0(21.0-36.0)$ & $35.0(30.0-42.5)$ & $20.0(17.0-22.5)$ & $<0.001$ \\
\hline PCWP, mmHg & $20.0 \pm 9.0$ & $25.9 \pm 6.8$ & $11.8 \pm 3.9$ & $<0.001$ \\
\hline Cardiac index, ml/min/m2 & $2.2 \pm 0.6$ & $2.0 \pm 0.6$ & $2.4 \pm 0.4$ & 0.001 \\
\hline LVEDP, mmHg & $20.0(14.5-27.5)$ & $24.5(21.0-30.7)$ & $15.0(13.0-17.5)$ & $<0.001$ \\
\hline SVR, dyn $\times \sec \times \mathrm{cm}^{-5}$ & $1619.5(1345-2029.0)$ & $1712.0(1344-2178.0)$ & $1536.0(1334-1794.0)$ & 0.189 \\
\hline PVR, dyn $\times \sec \times \mathrm{cm}^{-5}$ & $205.5(138.2-251.0)$ & $212.0(164.5-288.5)$ & $180.0(111.0-238.5)$ & 0.056 \\
\hline
\end{tabular}

Data are expressed as mean $\pm \mathrm{SD}$, median with IQR, or absolute numbers and respective percentages. $\mathrm{PH}$, pulmonary hypertension; RA, right atrial; RV, right ventricular; RVED and RVES, RV end diastolic and end systolic; FAC, fractional area change; TAPSE, tricuspid annular plane systolic excursion; RV-S', RV peak systolic velocity; LV-GLPS, left ventricular global longitudinal peak systolic strain; LVEDP, left ventricular enddiastolic pressure; mPAP, mean pulmonary artery pressure; PCWP, pulmonary capillary wedge pressure; SVR, systemic vascular resistance; PVR, pulmonary vascular resistance

RV strain) and invasively assessed mPAP and PCWP in patients with predominantly post-capillary $\mathrm{PH}$, while no significant associations were found in non-PH patients. To our knowledge, this is the first study providing a comprehensive analysis of right heart strain parameters in comparison with invasive hemodynamic measurements in patients with predominantly post-capillary $\mathrm{PH}$.

Imaging of the right heart, especially of the RA, by TTE often remains challenging. Since we mainly examined patients with post-capillary $\mathrm{PH}$ which also have several left heart and valve diseases, imaging of the right heart can become even more challenging. Still 2D TTE remains the most widely accessible and used diagnostic tool in cardiology and additional methods like STE are needed to extend the information derived from TTE in different clinical settings. Several studies such as Padeletti et al. [9] and Peluso et al. [10] have shown that STE is a valuable and practicable method for RA and RV function assessment.

$\mathrm{PH}$ causes an increase of RV afterload with consecutive elevation of RV pressures, which require adaptation methods of the RV such as intensifying contraction and increasing muscle mass (myocardial hypertrophy). This can result in impairment of RV systolic function, making parameters for RV systolic function such as RVS some of the most valuable predictors for outcome in patients with PH [20]. Previous studies [13, 14, 21] reported impaired RA and RV strain values in patients with precapillary PH. Similarly, in our study RA strain values were lower and RV strain values higher in $\mathrm{PH}$ patient compared to non-PH patient. While $\mathrm{PH}$ patients in our study consisted of an all-comer cohort and mainly represented patients with post-capillary $\mathrm{PH}$ caused by left heart diseases, previous studies that reported significant

Table 3 Right atrial (RA) and right ventricular (RV) strain parameters

\begin{tabular}{|c|c|c|c|c|}
\hline Parameter & All $(n=78)$ & $\mathrm{PH}(\mathrm{n}=45)$ & Non-PH $(n=33)$ & $p$-value \\
\hline \multicolumn{5}{|l|}{ RA strain parameters } \\
\hline RAS, $\%$ & $19.5(10.4-26.9)$ & $14.8(7.5-26.3)$ & $21.3(15.3-29.9)$ & 0.060 \\
\hline Conduit function, \%* & $10.0(5.8-14.7)$ & $7.5(3.7-14.7)$ & $11.7(7.6-15.6)$ & 0.063 \\
\hline Contractile function, $\%^{*}$ & $11.8(8.4-15.8)$ & $11.1(5.3-13.9)$ & $13.5(9.2-16.5)$ & 0.870 \\
\hline \multicolumn{5}{|l|}{ RV strain parameters } \\
\hline RVS, \% & $-12.3 \pm 6.5$ & $-11.5 \pm 6.3$ & $-13.6 \pm 6.8$ & 0.196 \\
\hline Basal strain, \% & $-21.4 \pm 9.3$ & $-20.4 \pm 10.7$ & $-23.0 \pm 6.1$ & 0.210 \\
\hline Mid strain, \% & $-19 \pm 7.3$ & $-17.4 \pm 7.8$ & $-21.6 \pm 5.5$ & 0.019 \\
\hline Apical strain, \% & $-9.3(-16.9--5.9)$ & $-8.8(-15.2--5.8)$ & $-11.1(-18.7--6.6)$ & 0.350 \\
\hline Free Wall, \% & $-17.2 \pm 6.5$ & $-16.15 \pm 7.1$ & $-19.0 \pm 4.8$ & 0.072 \\
\hline
\end{tabular}

Data are expressed as mean \pm SD or median with IQR. $\mathrm{PH}$, pulmonary hypertension; RAS, mean right atrial reservoir strain; RVS, mean right ventricular strain; ${ }^{*}=$ atrial fibrillation, RA conduit and contraction function was only calculated for 59 patients $(\mathrm{PH} n=30$, non- $\mathrm{PH} n=29)$. 
Table 4 Correlation analysis of mean right atrial (RA) and right ventricular (RV) strain parameters with invasively obtained hemodynamic parameters

\begin{tabular}{|c|c|c|c|c|c|c|c|c|}
\hline & $\mathrm{PH}(\mathrm{n}=45)$ & & & & Non-PH $(n=33)$ & & & \\
\hline \multirow[t]{2}{*}{ Parameter } & mPAP & & PCWP & & mPAP & & PCWP & \\
\hline & $\begin{array}{l}\text { correlation } \\
\text { coefficient }\end{array}$ & $\begin{array}{l}p \\
\text { value }\end{array}$ & $\begin{array}{l}\text { correlation } \\
\text { coefficient }\end{array}$ & $\begin{array}{l}\mathrm{p} \\
\text { value }\end{array}$ & $\begin{array}{l}\text { correlation } \\
\text { coefficient }\end{array}$ & $\begin{array}{l}\mathrm{p} \\
\text { value }\end{array}$ & $\begin{array}{l}\text { correlation } \\
\text { coefficient }\end{array}$ & $\begin{array}{l}\mathrm{p} \\
\text { value }\end{array}$ \\
\hline RA area & 0.297 & 0.050 & 0.189 & 0.219 & 0.043 & 0.814 & 0.281 & 0.126 \\
\hline RVED area & 0.382 & 0.012 & 0.389 & 0.010 & 0.106 & 0.598 & 0.142 & 0.489 \\
\hline RVES area & 0.271 & 0.078 & 0.338 & 0.027 & -0.026 & 0.899 & 0.026 & 0.900 \\
\hline FAC & 0.089 & 0.572 & 0.130 & 0.407 & 0.157 & 0.434 & 0.031 & 0.882 \\
\hline RV base diameter & 0.346 & 0.023 & 0.318 & 0.038 & 0.051 & 0.792 & 0.213 & 0.277 \\
\hline RV mid diameter & 0.251 & 0.104 & 0.188 & 0.227 & -0.182 & 0.343 & 0.182 & 0.355 \\
\hline $\begin{array}{l}\text { RV apex-base } \\
\text { diameter }\end{array}$ & 0.077 & 0.626 & 0.104 & 0.507 & -0.073 & 0.705 & -0.258 & 0.184 \\
\hline TAPSE & 0.378 & 0.010 & 0.181 & 0.233 & 0.245 & 0.154 & 0.310 & 0.085 \\
\hline$R V-S^{\prime}$ & 0.323 & 0.037 & 0.166 & 0.294 & 0.453 & 0.10 & -0.069 & 0.717 \\
\hline RVOT VTI & -0.364 & 0.019 & -0.222 & 0.16 & 0.377 & 0.044 & 0.059 & 0.77 \\
\hline$R V-e^{\prime}$ & 0.200 & 0.20 & 0.132 & 0.40 & 0.455 & 0.009 & 0.269 & 0.14 \\
\hline RV-a' & -0.455 & 0.009 & $-0,275$ & 0.13 & $-0,104$ & 0.58 & -0.111 & 0.56 \\
\hline \multicolumn{9}{|l|}{ RA strain parameters } \\
\hline RAS & -0.470 & 0.001 & -0.296 & 0.048 & -0.017 & 0.923 & -0.177 & 0.334 \\
\hline Conduit function * & -0.342 & 0.065 & -0.171 & 0.366 & 0.259 & 0.175 & -0.019 & 0.924 \\
\hline Contractile function * & -0.335 & 0.071 & -0.242 & 0.198 & 0.045 & 0.818 & -0.018 & 0.927 \\
\hline \multicolumn{9}{|l|}{ RV strain parameters } \\
\hline RVS & 0.490 & 0.001 & 0.365 & 0.015 & -0.130 & 0.520 & -0.052 & 0.802 \\
\hline Basal strain & 0.395 & 0.009 & 0.131 & 0.404 & 0.062 & 0.763 & -0.307 & 0.135 \\
\hline mid strain & 0.443 & 0.003 & 0.260 & 0.092 & -0.180 & 0.378 & -0.024 & 0.910 \\
\hline Apical strain & 0.461 & 0.002 & 0.348 & 0.220 & -0.298 & 0.139 & 0.242 & 0.244 \\
\hline Free Wall & 0.466 & 0.002 & 0.258 & 0.094 & -0.277 & 0.265 & -0.061 & 0.773 \\
\hline
\end{tabular}

$\mathrm{PH}$, pulmonary hypertension; mPAP, mean pulmonary artery pressure; PCWP, pulmonary capillary wedge pressure; RA, right atrial; RV, right ventricular; RVED and RVES, RV end diastolic and end systolic; FAC, fractional area change; TAPSE, tricuspid annular plane systolic excursion; RV-S', RV peak systolic velocity; RAS, mean right atrial reservoir strain; ${ }^{*}=$ atrial fibrillation, $\mathrm{RA}$ conduit and contraction function was only calculated for 59 patients $(\mathrm{PH} n=30, \mathrm{non}-\mathrm{PH} \mathrm{n}=29)$; $\mathrm{RVS}=$ mean right ventricular strain

impairment of RA and RV strain mainly focused on patients with idiopathic or scleroderma-associated $\mathrm{PH}[13]$ and/or chronic thromboembolic pulmonary hypertension $[14,21]$. Precapillary PH may cause an earlier onset and more severe impairment of right heart function. This makes comparisons of these studies with our findings difficult as individual entities of $\mathrm{PH}$ and different comorbidities may have a specific impact on RA and RV function.

Standard echocardiographic right heart function parameters used in clinical routine comprise TAPSE, RV-S ', or FAC. In our study TAPSE and RV-S' showed a significant difference between $\mathrm{PH}$ and non-PH patients with lower levels for both parameters in the presence of $\mathrm{PH}$ while RA and RV strain parameters did not differ between $\mathrm{PH}$ and non-PH patients. Still there was a trend towards lower RAS and RV free wall levels in $\mathrm{PH}$ patients which should be validated in a larger sample size. Concerning associations with hemodynamic parameters (mPAP and PCWP), RAS and RVS showed stronger associations in patients with $\mathrm{PH}$ compared to TAPSE and RV-S'. Other studies recently described that 2D STE of the RV free wall has been shown to be a feasible and more accurate parameter for predicting outcome and cardiovascular events in patients with predominantly pre-capillary PH $[11,21,22]$. This superiority is mainly explained by the global assessment of right heart function by 2D STE and higher sensitivity of 2D STE while e.g. TAPSE remains a localized, onedimensional measurement of the tricuspid annular motion [21, 22]. This suggests that right heart 2D STE may be a more accurate method to detect functional myocardial impairment compared to standard echocardiographic parameters. 
Long-term pressure overload and adaptation due to LV dysfunction and $\mathrm{PH}$ may result in RV diastolic dysfunction and affect the pressure gradient between RA and RV [5, 23], which leads to RA remodeling, enlargement and dysfunction [24]. Assessment of RA function can be implemented in TTE by using 2D STE. In our study, RAS showed a moderate inverse association with $\mathrm{mPAP}$ and a weak inverse correlation with PCWP in patients with $\mathrm{PH}$. Regarding RA conduit and contractile function, associations with mPAP and PCWP did not reach statistical significance, which may be a result of the small sample size due to the exclusion of AF patients for these two parameters. The reported moderate associations of RAS and RVS with invasive pulmonary pressures may be explainable by the fact that RAS and RVS are, next to an increase of RV afterload, significantly associated with geometric and structural properties of the RA and the RV (such as hypertrophy and remodeling processes) with impact on compliance and wall tension, as well as on systolic RV and LV performance [8, 25]. The results of our study, however, demonstrate that RAS may be a valuable noninvasive parameter for monitoring right heart function of $\mathrm{PH}$ patients. Sato et al. [3] and Alenezi et al. [5] have shown that RA reservoir, conduit and contractile function are independent predictors for mortality and hospitalization in patients with PH (WHO group 1 and 4). Further studies are wanted to validate whether the predictive value of RA function parameters is also applicable in patients with post-capillary $\mathrm{PH}$.

\section{Limitations}

The present study has several limitations. First, it is a single-center analysis with a relatively small patient cohort; while there were significant associations with invasively and non-invasively assessed parameters, correlation strength and significance may be underestimated.

Second, our cohort consisted of all-comers of the cardiology department and many patients had multiple other cardiovascular diseases besides $\mathrm{PH}$, which may have a relevant impact on the results. On the contrary, this makes our findings more applicable and suitable for daily clinical routine. Since the etiology of PH in our patient cohort was predominantly post-capillary, these results might not be applicable for pre-capillary $\mathrm{PH}$. As mentioned above, most of previous studies in this field focussed on precapillary $\mathrm{PH}$ excluding patients with left heart diseases. While long-term LV dysfunction may finally lead to post-capillary $\mathrm{PH}$, it may take longer to cause measurable functional impairments of the RA and $\mathrm{RV}$ and some of our patients might not even have reached that point yet $[26,27]$.

Third, this study did not include follow-up measurements. Future studies are needed to investigate the prognostic value of 2D STE derived RA and RV parameters for mortality and long-term outcome in patients with post-capillary $\mathrm{PH}$.

\section{Conclusion}

Our study showed significant associations between invasively assessed hemodynamic parameters and right heart strain parameters, particularly RAS and RVS, in patients with predominantly post-capillary $\mathrm{PH}$. The analysis of RA and RV strain may be useful for monitoring of right heart function in these patients. Prospective studies evaluating RA and RV strain alterations in patients with different entities of $\mathrm{PH}$ are needed to clarify the prognostic value of these parameters.

\section{Supplementary information}

Supplementary information accompanies this paper at https://doi.org/1 0.1186/s12947-020-00197-z

Additional file 1: Table S1. Associations of diverse clinical characteristics and RV strain with $\mathrm{PH}$; multivariate regression analysis. Table S2. Associations of diverse clinical characteristics and RA strain with $\mathrm{PH}$; multivariate regression analysis.

\section{Abbreviations}

RA: Right atrium / atrial; RV: Right ventricle / ventricular; 2D STE: 2D speckle tracking echocardiography; PH: Pulmonary hypertension; mPAP: Mean pulmonary arterial pressure; PCWP: Pulmonary capillary wedge pressure; RAS: Average peak systolic right atrial strain / right atrial reservoir function; RVS: Average peak systolic right ventricular strain; PAP: Pulmonary artery pressure; TTE: Transthoracic echocardiography; TAPSE: Tricuspid annular plane systolic excursion; RV-S': Right ventricle peak systolic velocity; DTI: Doppler tissue imaging; ASE: Amercian Society of Echocardiography; EACVI: European Association of Cardiovascular Imaging; RVED area: Right ventricular end-diastolic area; RVES area: Right ventricular end-systolic area; FAC: Right ventricle fractional area change; LVEF: Left ventricular ejection fraction; LV: Left ventricle; ROI: Region of interest; LV-GLPS: Left ventricular global longitudinal peak systolic strain; LVEDP: Left ventricular end-diastolic pressure; SVR: Systemic vascular resistance; PVR: Pulmonary vascular resistance; ICC: Intraclass correlation coefficient; SD: Standard deviation; IQR: Interquartile ranges; Non-PH: Patients without pulmonary hypertension; RVES area: Right ventricular end-systolic area; Cl: Confidence interval; CTEPH: Chronic thrombembolic pulmonary hypertension

\section{Acknowledgements}

B.H. was a member of the BIH Charité Clinician Scientist Program sponsored by the Charité - Universitätsmedizin Berlin and the Berlin Institute of Health $(\mathrm{BIH})$.

\section{Authors' contributions}

$\mathrm{AB}, \mathrm{BH}$ and FK: study conception and design, data acquisition, data analysis, interpretation of data, critical revision of the manuscript, approval of article; LT: data acquisition, data analysis, interpretation of data, drafting of the manuscript, approval of article; KS and HD: data acquisition, data analysis, interpretation of data, critical revision of the manuscript, approval of article; AH: data acquisition, critical revision of the manuscript, approval of article.

\section{Funding}

None.

\section{Availability of data and materials}

The data that support the findings of this study are available from the corresponding author upon reasonable request.

\section{Ethics approval and consent to participate}

The study has been approved by the institutional ethics committee of the Charité-Universitätsmedizin Berlin (EA4/067/13) and complied with the Declaration of Helsinki. All examinations had a clinical indication and patients gave their consent on using their anonymized data for study purposes. 


\section{Consent for publication}

Not applicable.

\section{Competing interests}

The authors declare that they have no competing interests.

\section{Author details}

${ }^{1}$ Medizinische Klinik m.S. Kardiologie und Angiologie,

Charité-Universitätsmedizin, Campus Mitte, Charitéplatz 1, 10117 Berlin, Germany. ${ }^{2}$ DZHK (German Center for Cardiovascular Research), partner site, Berlin, Germany. ${ }^{3}$ Berlin Institute of Health (BIH), Berlin, Germany. ${ }^{4}$ Zentrum für Kardiologie, Kardiologische Gemeinschaftspraxis, Muenster, Germany. ${ }^{5}$ Department of Cardiology III - Adult Congenital and Valvular Heart Disease, University Hospital Muenster, Muenster, Germany.

Received: 22 February 2020 Accepted: 6 May 2020

Published online: 14 May 2020

\section{References}

1. Galie N, Humbert M, Vachiery JL, Gibbs S, Lang I, Torbicki A, et al. 2015 ESC/ ERS guidelines for the diagnosis and treatment of pulmonary hypertension. Rev Esp Cardiol (Engl Ed). 2016;69(2):177.

2. Haeck ML, Scherptong RW, Marsan NA, Holman ER, Schalij MJ, Bax JJ, et al. Prognostic value of right ventricular longitudinal peak systolic strain in patients with pulmonary hypertension. Circ Cardiovasc Imaging. 2012;5(5): 628-36

3. Sato T, Tsujino I, Ohira H, Oyama-Manabe N, Ito YM, Yamada A, et al. Right atrial volume and reservoir function are novel independent predictors of clinical worsening in patients with pulmonary hypertension. J Heart Lung Transplant. 2015;34(3):414-23.

4. Querejeta Roca G, Campbell P, Claggett B, Solomon SD, Shah AM. Right Atrial Function in Pulmonary Arterial Hypertension. Circ Cardiovasc Imaging. 2015;8(11):e003521; discussion e.

5. Alenezi F, Mandawat A, II'Giovine ZJ, Shaw LK, Siddiqui I, Tapson VF, et al. Clinical utility and prognostic value of right atrial function in pulmonary hypertension. Circ Cardiovasc Imaging. 2018;11(11):e006984.

6. Brand A, Bathe M, Oertelt-Prigione S, Seeland U, Rucke M, Regitz-Zagrosek V, et al. Right heart function in impaired left ventricular diastolic function: $2 \mathrm{D}$ speckle tracking echocardiography-based and Doppler tissue imagingbased analysis of right atrial and ventricular function. Echocardiography. 2018;35(1):47-55.

7. Padeletti M, Cameli M, Lisi M, Zaca V, Tsioulpas C, Bernazzali S, et al. Right atrial speckle tracking analysis as a novel noninvasive method for pulmonary hemodynamics assessment in patients with chronic systolic heart failure. Echocardiography. 2011;28(6):658-64.

8. Brand A, Bathe M, Hubscher A, Baldenhofer G, Hattasch R, Seeland U, et al. Normative reference data, determinants, and clinical implications of right atrial reservoir function in women assessed by 2D speckle-tracking echocardiography. Echocardiography. 2018.

9. Padeletti M, Cameli M, Lisi M, Malandrino A, Zaca V, Mondillo S. Reference values of right atrial longitudinal strain imaging by two-dimensional speckle tracking. Echocardiography. 2012;29(2):147-52.

10. Peluso D, Badano LP, Muraru D, Dal Bianco L, Cucchini U, Kocabay G, et al. Right atrial size and function assessed with three-dimensional and speckletracking echocardiography in 200 healthy volunteers. Eur Heart J Cardiovasc Imaging. 2013;14(11):1106-14.

11. Motoji Y, Tanaka H, Fukuda $Y$, Ryo K, Emoto N, Kawai H, et al. Efficacy of right ventricular free-wall longitudinal speckle-tracking strain for predicting long-term outcome in patients with pulmonary hypertension. Circ J. 2013; 77(3):756-63.

12. Fukuda $Y$, Tanaka $H$, Sugiyama $D$, Ryo $K$, Onishi $T$, Fukuya $H$, et al. Utility of right ventricular free wall speckle-tracking strain for evaluation of right ventricular performance in patients with pulmonary hypertension. J American Soc Echocardiography. 2011;24(10):1101-8.

13. Bhave NM, Visovatti SH, Kulick B, Kolias TJ, McLaughlin W. Right atrial strain is predictive of clinical outcomes and invasive hemodynamic data in group 1 pulmonary arterial hypertension. Int J Cardiovasc Imaging. 2017;33(6):847-55.

14. Fukuda $Y$, Tanaka H, Ryo-Koriyama K, Motoji $Y$, Sano H, Shimoura $H$, et al. Comprehensive functional assessment of right-sided heart using speckle tracking strain for patients with pulmonary hypertension. Echocardiography. 2016;33(7):1001-8.

15. Rudski LG, Lai WW, Afilalo J, Hua L, Handschumacher MD, Chandrasekaran K, et al. Guidelines for the echocardiographic assessment of the right heart in adults: a report from the American Society of Echocardiography endorsed by the European Association of Echocardiography, a registered branch of the European Society of Cardiology, and the Canadian Society of Echocardiography. Journal of the American Society of Echocardiography: official publication of the American Society of Echocardiography. 2010;23(7): 685-713; quiz 86-8.

16. Lang RM, Badano LP, Mor-Avi V, Afilalo J, Armstrong A, Ernande L, et al. Recommendations for cardiac chamber quantification by echocardiography in adults: an update from the American Society of Echocardiography and the European Association of Cardiovascular Imaging. Euro Heart J Cardiovasc Imaging. 2015;16(3):233-71.

17. Hewing B, Theres L, Spethmann S, Stangl K, Dreger H, Knebel F. Left atrial strain predicts hemodynamic parameters in cardiovascular patients. Echocardiography.n/a-n/a.

18. Voigt JU, Pedrizzetti G, Lysyansky P, Marwick TH, Houle H, Baumann R, et al. Definitions for a common standard for 2D speckle tracking echocardiography: consensus document of the EACVI/ASE/industry task force to standardize deformation imaging. Eur Heart J Cardiovasc Imaging. 2015;16(1):1-11.

19. Badano LP, Kolias TJ, Muraru D, Abraham TP, Aurigemma G, Edvardsen T, et al. Standardization of left atrial, right ventricular, and right atrial deformation imaging using two-dimensional speckle tracking echocardiography: a consensus document of the EACVI/ASE/industry task force to standardize deformation imaging. Eur Heart J Cardiovasc Imaging. 2018;19(6):591-600.

20. Ghio S, Klersy C, Magrini G, D'Armini AM, Scelsi L, Raineri C, et al. Prognostic relevance of the echocardiographic assessment of right ventricular function in patients with idiopathic pulmonary arterial hypertension. Int J Cardiol. 2010;140(3):272-8.

21. Fine NM, Chen L, Bastiansen PM, Frantz RP, Pellikka PA, Oh JK, et al. Outcome prediction by quantitative right ventricular function assessment in 575 subjects evaluated for pulmonary hypertension. Circ Cardiovasc Imaging. 2013;6(5):711-21.

22. Fukuda Y, Tanaka H, Motoji Y, Ryo K, Sawa T, Imanishi J, et al. Utility of combining assessment of right ventricular function and right atrial remodeling as a prognostic factor for patients with pulmonary hypertension. Int J Cardiovasc Imaging. 2014;30(7):1269-77.

23. Bristow MR, Zisman LS, Lowes BD, Abraham WT, Badesch DB, Groves BM, et al. The pressure-overloaded right ventricle in pulmonary hypertension. Chest. 1998;114(1 Suppl):101S-6S.

24. Willens HJ, Fertel DP, Qin J, Labrador E, Lowery MH. Effects of age and pulmonary arterial hypertension on the different phases of right atrial function. Int J Cardiovasc Imaging. 2008;24(7):703-10.

25. Lee $\mathrm{JH}$, Park JH. Strain analysis of the right ventricle using two-dimensional echocardiography. J Cardiovasc Imaging. 2018;26(3):111-24.

26. Vachiery JL, Adir Y, Barbera JA, Champion H, Coghlan JG, Cottin V, et al. Pulmonary hypertension due to left heart diseases. J Am Coll Cardiol. 2013; 62(25 Suppl):D100-8.

27. Fang JC, DeMarco T, Givertz MM, Borlaug BA, Lewis GD, Rame JE, et al. World Health Organization pulmonary hypertension group 2: pulmonary hypertension due to left heart disease in the adult--a summary statement from the pulmonary hypertension Council of the International Society for heart and lung transplantation. J Heart Lung Transplant. 2012;31(9):913-33.

\section{Publisher's Note}

Springer Nature remains neutral with regard to jurisdictional claims in published maps and institutional affiliations. 